\title{
ROSA CHACEL: NOVELISTA Y TRADUCTORA ESPAÑOLA EXILIADA
}

\author{
Lieve Behiels \\ Katholieke Universiteit Leuven, Facultad de Letras. Leuven, Belgium.
}

\begin{abstract}
Resumen: Rosa Chacel pasó varias décadas en el exilio tras la guerra civil española. Residió principalmente en Buenos Aires y en Río de Janeiro. Durante este período de su vida combinó su obra de novelista y ensayista con traducciones literarias. En este artículo vamos a presentar la labor traductora de Rosa Chacel desde varios ángulos. En primer lugar, nos interesaremos por las circunstancias de su trabajo de traductora. También nos preguntaremos hasta qué punto dominaba Rosa Chacel las lenguas extranjeras de las que traducía. Luego veremos qué huellas ha dejado esta actividad en su obra novelística. Finalmente, nos preguntaremos qué puentes se pueden tender entre las obras que traduce y sus propias concepciones estéticas.
\end{abstract}

Palabras clave: Rosa Chacel. Exilio. Traducción Literaria. Creación.

\section{ROSA CHACEL: SPANISH NOVELIST AND TRANSLATOR IN EXILE}

\begin{abstract}
Rosa Chacel spent several decades in exile after the Spanish civil war. She lived mainly in Buenos Aires and Rio de Janeiro. During this period of her life she combined her work as a novelist and essayist with literary translations. In this article we will present the work of Rosa Chacel as a translator from various angles. First, we examine the circumstances of her work as a translator. Our next question is to what extent Rosa Chacel dominated the foreign languages she was translating from. Then we will look at the traces this activity left in her novels. Finally, we consider the connections that can be established between the works she translated and her own aesthetic concepts.
\end{abstract}

Key words: Rosa Chacel. Exile. Literary translation. Creation. 


\section{Introducción}

En 1936, Rosa Chacel era una joven escritora de vanguardia que tenía en su haber, entre otras, una novela, Estación. Ida y vuelta (1930) y varias contribuciones en la revista cultural y literaria más prestigiosa del momento, la Revista de Occidente, dirigida por Ortega y Gasset. Vivía en Madrid y había pasado una temporada en Roma, entre 1922 y 1927 (Chacel 1980, 33), además de una estancia de varios meses en Berlín en 1933. Durante la guerra civil, la autora publicó varias contribuciones en revistas republicanas (Rodríguez Fischer 1990, 116). Después de varios meses en Valencia, donde colaboró con la revista Hora de España, se trasladó a París en marzo de 1937. De allí pasó a Grecia en 1938 donde volvió a encontrarse con el autor Nikos Kazantzakis que había conocido en París. A principios de 1939 se reunió con su marido en Ginebra. Tanto ella como su marido se habían significado en los años de la Guerra Civil por actividades de apoyo a la República, por lo cual no tenían ningún futuro en la España de Franco. Estando en Suize surgió el proyecto de ir a América (Chacel 1980, 48). Llegaron a Río de Janeiro el 4 de junio de 1940. Rosa Chacel proyectó ir a Buenos Aires para que su hijo pudiera escolarizarse en español, así como para reanudar su carrera literaria, ya que tendría más oportunidades en Argentina. Además, Guillermo de Torre le ofreció publicar allí su novela Teresa (ibidem, 56). El libro saldría publicado en 1941 en la editorial Nuevo Romance. Esta editorial, como otras en las que publicó, fue fundada por exiliados españoles: Rafael Dieste, Rafael Alberti y Francisco Ayala (Matamoro 2009, 79). A partir de 1942 la autora se instaló en Buenos Aires, pasando temporadas frecuentes en Río.

Aunque las autoridades argentinas no se mostraron realmente entusiasmadas ante la llegada de exiliados de España ${ }^{1}$, Buenos

\footnotetext{
${ }^{1}$ Como observa Nora Pasternac, "[e]n Argentina, el gobierno no sólo no favoreció la llegada de los republicanos sino que sus representantes se inclinaron más bien por el triunfo del franquismo sin grandes problemas de conciencia: no rompieron relaciones con los usurpadores y aceptaron inmediatamente la instalación oficial de la embajada del nuevo régimen" $(2005,9)$.
}

Cad. Trad., Florianópolis, v. 38, $\mathrm{n}^{0}$ 1, p. 47-64, jan-abr, 2018 
Aires constituía para ellos un contexto interesante para poder continuar su labor. Argentina vivía los comienzos del auge de su industria cultural (Willson 2009, 233). Se establecieron nuevas casas editoriales, algunas fundadas por españoles: en 1938, Gonzalo Losada, español republicano, fundó Losada donde publicó la obra de muchos exiliados (ibidem, 241). Antonio López Llausás, otro exiliado republicano, puso en marcha Sudamericana, que dio a conocer numerosos autores nuevos y se caracterizó por la calidad de sus traducciones (Lago Carballo \& Gómez-Villegas 2009, 129). Rosa Chacel publicó libros propios y traducciones en ambas casas, así como en Emecé, otra editorial fundada por republicanos exiliados, gallegos en este caso. Victoria Ocampo había fundado en 1931 la revista Sur, inspirada por la Revista de Occidente de Ortega (King 2009, 176) y abría la publicación a numerosos escritores del exilio español, entre otros a la propia Rosa Chacel (Macciuci 2004, 54). Además, todos los secretarios de redacción de Sur también se dedicaban a la traducción (Willson 2009, 240), una muestra más de la apertura internacional de la revista. Entre octubre de 1939 y enero de 1960, Chacel publicó 23 textos en Sur aunque su relación con la revista pasó por altibajos de los que dan cuenta sus diarios y que tienen que ver más que nada con la tensión entre la autora y la directora de la revista ${ }^{2}$. Además, escribió para las revistas Realidad - fundada por los exiliados españoles Lorenzo Luzariaga y Francisco Ayala (Otero Carvajal 2006, 129) - y Anales de Buenos Aires, así como para el diario La Nación ${ }^{3}$.

\footnotetext{
${ }^{2}$ Carmen Morán Rodríguez presenta el problema que significaba Ocampo para Chacel en los siguientes términos: "Victoria Ocampo era, con dramática exactitud, su opuesto: rica y desenvuelta - tan rica y tan desenvuelta que en su caso la falta de estudios universitarios no tenía importancia-, agraciada y —-sobre todo-sofisticada, rutilante anfitriona de eventos sociales en los que Chacel nunca sabía comportarse con habilidad" $(2013,201)$.

${ }^{3}$ Estas contribuciones que comprenden poemas, ensayos, relatos de ficción y crítica literaria se han publicado en los volúmenes III y IV de las Obras completas de Rosa Chacel.
} 


\section{2. ¿Qué significa traducir?}

A continuación, vamos a presentar la labor traductora de Rosa Chacel desde varios ángulos. En primer lugar, nos interesaremos por las circunstancias de su trabajo de traductora. También nos preguntaremos hasta qué punto dominaba Rosa Chacel las lenguas extranjeras de las que traducía. Luego veremos qué huellas ha dejado esta ocupación en su obra novelística. Finalmente, analizaremos qué puentes se pueden tender entre las obras que traduce y sus propias concepciones estéticas.

Para Rosa Chacel, como para muchos autores en la misma situación ${ }^{4}$, la traducción constituía uno de los medios para ganar algún dinero y para seguir en contacto con el mundo editorial, con vistas a la publicación de sus propias obras. La primera parte de sus diarios publicados bajo el título Alcancía. Ida, correspondiente a los años 1940-1960, proporciona alguna información sobre cómo enfocaba la autora su labor traductora. Teniendo en cuenta que, salvo una sola anotación en 1940, no escribe en el diario hasta 1952, no debe sorprendernos que no aparezca ninguna mención a su traducción de La peste de Camus, publicada en 1948 por Emecé y autorizada por la censura española en 1957 (Cruces Colado 2006, 96), con numerosas reediciones. Los apuntes del diario relacionados con la traducción suelen ser breves incisos, como el siguiente, correspondiente al 4 de agosto de 1954: "Por la noche leí Sur, que acababa de llegar, luego trabajé un poco en la endiablada traducción y no fui capaz de continuar lo empezado aquí" (Chacel 1982, 34). No menciona qué traducción tiene entre manos, podría tratarse, por la fecha, de una obra de teatro de Christopher Fry, La dama no es para la hoguera o Venus observada que se publicarían en 1955 en la editorial Sudamericana. Lo que considera "su trabajo" no incluye realmente la traducción, como pone de manifiesto este apunte del 25 de diciembre de 1956:

\footnotetext{
${ }^{4}$ El panorama de los traductores españoles exiliados activos en Argentina ha sido analizado en detalle en la tesis doctoral de Germán Loedel Rois. Para los datos relacionados con Rosa Chacel véase Loedel Rois $(2012,226)$.
}

Cad. Trad., Florianópolis, v. 38, n $^{0}$ 1, p. 47-64, jan-abr, 2018 
Bueno, en todo este tiempo han llovido actividades: nada me interesa porque nada de ello ha resuelto mi situación económica. Por supuesto, he podido seguir tirando, pero sacrificando mi trabajo íntegramente. Traducciones, conferencias [...]. (Ibidem, 67)

La traducción no pasa de ser, pues, una mera 'actividad'. Dos días más tarde apunta una serie de cosas que le quedan por hacer: "Ahora tengo que reunir los materiales de Sor Juana y terminar Berenice, escribir a Fernanda, escribir a Concha, escribir a Timo largamente..." (Ibidem, 71). La traducción de una obra de Racine queda intercalada entre la preparación de una conferencia ${ }^{5}$ y la correspondencia con su esposo y con sus amigas Fernanda Monasterio y Concha de Albornoz. La traducción de Berenice saldría publicada junto con otras dos obras de Racine en 1958 pero apenas la menciona de pasada.

En 1957 realiza la traducción de un libro de su amigo Nikos Kazantzakis, Libertad o muerte. De la lectura del diario se desprende que se imponía un ritmo de trabajo riguroso: el sábado 6 de abril apunta: "Al mismo tiempo hice el número de páginas que me había propuesto de la traducción y leí unos cuantos libros" (ibidem, 85), y al día siguiente: "Anoche trabajé hasta tarde; me traduje diez páginas y leí dos libros” (85). Más que el traducir le cuesta el reanudar el contacto epistolar con Kazantzakis y su esposa, con los que tenía lazos de amistad desde los años treinta: “¿Seré capaz de escribir a Maruja y a Kazantzakis? A éste debo absolutamente escribirle. Pero ¿cómo escribirle porque debo? Tantos, tantos años sin decir una palabra" (86). Mientras tanto, corrige las pruebas de la traducción de Berenice. Y unos días más tarde exclama: "No querría traducir más, pero veo que es imposible prescindir de eso" (89). El uno de mayo a las siete de la tarde termina la traducción de Libertad o muerte (96). Meses después, el 19 de julio, reflexio-

${ }^{5}$ Rosa Chacel pronunció una conferencia sobre Sor Juana en 1958 en la Universidad Nacional del Sur, Bahía Blanca, publicada bajo el título de "Poesía de circunstancia" (Chacel 1993b, 11-41).

Cad. Trad., Florianópolis, v. 38, n $^{0}$ 1, p. 47-64, jan-abr, 2018 
na sobre su cansancio: "El verano en Río ha sido horroroso; en gran parte porque la traducción del libro de Kazantzakis me agotó durante los primeros meses" (100). La traducción se publica el mismo año 1957.

El año siguiente traduce Phèdre de Racine e intenta publicarla por capítulos en Sur. Ahora le preocupa no tanto el encontrar un canal de publicación sino la posibilidad de recibir una remuneración correcta. El viernes 26 de agosto de 1958 anota: "Voy a ponerme a copiar la Fedra para ver si hay medio de publicarla en Sur. Bueno, publicarla es casi seguro, pero ¿cobrarla?..." (125). El 2 de enero de 1959 consigna que la traducción entera está terminada: "Tengo ahí la Fedra terminada y no es posible obtener los cuatro mil pesos que necesitaría" (138). A finales de ese mes escribe en el diario la cantidad que ha cobrado por la prepublicación: "Y además cobré el primer acto y prólogo de la Fedra, 180 pesos... Esto no se cree. Incluso tengo idea de que no está permitido: ahora esas cosas están más o menos legisladas y una cantidad como esa toca en lo delictivo" (147). Sin embargo no consta que haya protestado por haber cobrado tan poco.

Entre 1959 y 1961 la autora reside en Nueva York con una beca de la Fundación Guggenheim para escribir un ensayo, Saturnal, que publicaría finalmente en 1972. A partir de Nueva York hace un viaje a México y en 1962 vuelve por primera vez desde la guerra a España. En el verano de 1962 viaja a París donde permanece un año más, para volver a Río en 1963. Regresa a Río con un encargo de traducción de la editorial de la Revista de Occidente: Teoría del arte de vanguardia de Renato Poggioli.

La estancia en Francia no le parece del todo productiva, pero quiere seguir en Europa para fomentar la publicación de sus obras. El 29 de agosto de 1962, apunta en su diario: "Tengo la seguridad de que no podré trabajar hasta que esté allí, pero creo que debo aguantar hasta ver si sale algo en octubre, de París - traducciones - y de Madrid - reediciones - [...]" (Chacel 1982, 269). Aunque sus traducciones apenas le merecía observaciones de pasada, era muy consciente de la importancia que tenía para un autor ser traducido. 
Es pesimista con respecto a las posibilidades en este campo. El 20 de septiembre de 1965 escribe: "Otra cosa que pesa enormemente en mi ánimo es que no tuve respuesta de la persona que me anunció una posible traducción de Teresa al italiano. No, mis libros no tienen posibilidad de existir en Europa" (ibidem, 423). El 15 de diciembre de 1965 la editorial alemana Piper Verlag rechaza publicar una posible traducción de La sinrazón, su novela más importante en la que trabajó entre 1952 y 1958 y que se publicó en 1960. Comenta lo siguiente: "El libro no se traducirá porque creen que no tendrá venta en Alemania. Es, por supuesto, la cosa más grave que podía pasarme en el terreno intelectual; suficiente, suficientísima para estar desesperada [...]" (ibidem, 428). Hasta donde hayamos podido investigar, aún no existe traducción de esta novela al alemán.

\section{Lenguas}

En la lista de los libros traducidos por Chacel se encuentran títulos de autores de lengua inglesa, francesa, italiana, griega, alemana y portuguesa. Es obvio que tenía un excelente conocimiento del francés, la segunda lengua de los españoles cultos de principios del siglo XX, perfeccionado, en el caso de la autora, por el hecho de haber pasado varias temporadas en París. En esta lengua contaba con competencias suficientes para realizar traducciones literarias de calidad. Había pasado cinco años en Roma, con lo cual esta lengua tampoco le planteaba problemas de comprensión. El portugués lo dominaba gracias a más de dos décadas de estancias frecuentes en Brasil. Es probable que la traducción de Libertad o muerte de Kazantzakis sea una traducción indirecta a través de la traducción francesa, publicada en 1956. Chacel trabajó en la traducción española a lo largo de 1957.

La propia autora se declaraba nula en inglés. El libro que más le influyó, Portrait of the artist as a young men, de Joyce, lo leyó y releyó en la traducción española de Dámaso Alonso (ibidem, 430). Cuando le conceden una beca Guggenheim para pasar una tempo- 
rada en Nueva York, empieza a estudiar inglés, "con una torpeza indescriptible" (17 de junio de 1959, ibidem, 167). Sin embargo, para estas fechas, ya se habían publicado varias traducciones de obras teatrales de T.S. Eliot, Christopher Fry y J.B. Priestley con su firma. La obra de Eliot lleva un prólogo de Patrick Orpen Dudgeon, profesor de inglés, amigo de la autora y autor de varias colaboraciones en Sur. Dudgeon había publicado un estudio sobre Eliot en Sur en 1946 y se puede suponer que habría contribuido a solucionar eventuales problemas lingüísticos. En el caso de Priestley, se podría hablar de traducción cooperativa, puesto que en la portada aparece junto al nombre de Chacel el de Vera Macarow, traductora y colaboradora ocasional de Sur. En el caso de Christopher Fry, no se menciona una segunda mano. Cabe la posibilidad de que un primer traductor hiciera una versión inicial y que luego Rosa Chacel aportara correcciones estilísticas, pero esta hipótesis necesitaría investigarse más a fondo. La propia autora alude a "traducciones y arreglos de traducciones para algunas editoriales" (Chacel 1980, 62) sin dar más precisiones.

Asimismo, la traducción de la biografía de Rilke por Hans Egon Holthusen (1960) es el resultado de la colaboración con Nicolás Wendkheim. Chacel alude a esta cooperación en su diario el 29 de diciembre de 1958: "Ayer vino Niki y me trajo los poemas de Rilke para traducir. Lo malo es que él ha intentado darles forma y no están suficientemente al pie de la letra" (Chacel 1982, 129). Da a entender que lo que precisaba era una traducción literal para dar a los poemas la forma que a ella le parecía conveniente. No se esconde la dificultad de la tarea (ibidem, 136). Termina la traducción en junio de 1959, antes de salir para Nueva York.

A la autora le unían lazos de gran amistad con el poeta brasileño Walmir Ayala del que tradujo un libro de poemas, Museo de cámara. Ayala, a su vez, tradujo al portugués las Memorias de Leticia Valle. 


\section{Traducir y novelar}

En la década de los cincuenta, Rosa Chacel publica el mayor número de sus traducciones y al mismo tiempo progresa en su mayor empresa novelística: La sinrazón. En la novela, situada en Buenos Aires en los años treinta y cuarenta, aparece una exiliada republicana, Herminia que, como la autora, sobrevive gracias a un sinfín de tareas intelectuales: "El trabajo de Herminia no tenía horas fijas, traducía para una editorial de obras científicas, daba lecciones de todo lo que se presentase la ocasión y todavía tenía tiempo de publicar notas sobre libros en una revista" (Chacel 1989, 232), una lista de ocupaciones acordes con la parcela del campo cultural abierta a los exiliados.

Pero el personaje traductor más interesante se encuentra en su última novela, Ciencias naturales, publicada en 1988, cuando la autora tenía 90 años. Elena, contrafigura de Chacel en varias novelas semiautobiográficas, al llegar a Buenos Aires, sobrevive económicamente trabajando en una librería y traduciendo. Al darse cuenta de que necesita un par de zapatos, reflexiona: "En resumen, tendré que aceptar alguna traducción, cosa enojosa, si no es voluntaria" (Chacel 1988, 24). Un motivo para aceptar una traducción son las circunstancias en las que el trabajo se lleva a cabo: "Me dije: ¡indiscutible! una traducción es un trabajo tolerable porque su oficina es la soledad" (ibidem, 33).

Muchos traductores podrían reconocerse en este deseo de retirarse en soledad. Entre propuestas de "ensayos de medicina, de psicología, de pedagogía" surge La educación sentimental de Flaubert. El personaje, dado a la procrastinación, retrasa unos días la lectura del libro y se da cuenta de la dificultad de 'naturalizar' su contenido debido a la gran distancia psicológica que separa la época actual del siglo XIX:

Acometo la traducción, prosa exquisita, tan fácil de adaptar a la nuestra. Lo leo de corrido y me encuentro con lo inadaptable a nuestro tiempo. Es en los ratos del Estambul 
donde me quedo a veces meditando: paragonando lo que todos estamos hartos de saber: a dónde fue a parar lo sentimental educado... (ibidem, 36).

Como Elena ha podido elegir una obra maestra literaria, no le cuesta trabajo traducirla: "Ya van cincuenta páginas: es fácil o más bien es delicioso. Largo trabajo, debido a la soledad" (ibídem, 85). Avanzando en la novela la traductora llega a un fragmento en el que el protagonista está en un club revolucionario y allí encuentra largas frases en español. Se trata del primer capítulo de la tercera parte. Frédéric Moreau se pasea entre los acontecimientos de la revolución de 1848. Ha preparado un discurso para soltarlo en un club revolucionario pero le dan la palabra a un camarada de Barcelona que suelta una arenga en español (Flaubert 1969, 331). Lo que normalmente llena de orgullo a Elena, ahora le produce rechazo: queda aterrada al comprobar la decadencia sufrida por los gritos de libertad en el siglo que separa su época de la revolución de julio de 1848:

Las mismas frases grandiosas y burdas, una especie de eco que hubiera resonado de talud en talud, hasta quedar en un rumor grosero... habiendo sido un grito sublime. [...] Y las frases copiadas - con un siglo de anticipación - tal como se dijo, se gritó, se aulló en un desesperado alarido, que no supo imponerse, que no quedó como voz de los grandes, sino estertor de los... (Chacel 1988, 123).

Aquí el trabajo de la traducción ha pasado de una mera empresa intelectual a un enfrentamiento con el fracaso del ideal de libertad que llevó al personaje al exilio.

El paso del tiempo se refleja en los proyectos de traducción: al final de la novela duda si acometer la traducción de La route des Flandres (1960) de Claude Simon. La traductora apunta su apreciación del libro y las dificultades que la esperan: 
Es admirable, caótico y riguroso; muy a propósito para sumergirme en su misterioso furor: pasión, laberinto de un alma, soledad de un jefe en el horror bélico, realista... No sé si debo atreverme, es muy difícil, exige la perfección de un léxico que temo no poseer (ibidem, 221).

Finalmente rechaza la novela de Simon y acepta una traducción científica sobre psicoanálisis que consiste en 14 libros. Uno de los mejores amigos de Elena vuelve a España y a ella le aterra el clima de asfixia intelectual que él le describe. Compara la paciencia que necesitará para llevar a cabo la traducción proyectada con la que necesitan "los de allá, que están viviendo con la paciencia de los labriegos, pendientes de lo que les caiga del cielo. Treinta y tantos años esperando que caiga, iy no cae!" (Ciencias 224-225).

\section{Traducciones que sí son obras: resonancias}

El conjunto de traducciones más sólido de la autora lo constituyen las tragedias de Racine, las tres primeras publicadas en Buenos Aires en 1958 y un conjunto de seis en Madrid en 1983. Este proyecto de traducción lo emprendió Chacel por voluntad propia y lo asume como obra suya: "Traducir la obra de Racine al castellano es empresa de dificultad indiscutible, pero una vez que se la acomete, la tarea proporciona tal placer que desaparece la idea de dificultad" (Chacel 1993a, 199).

También se puede hablar de afinidad en el caso de Cocteau. Ya en 1928, la autora publicó una reseña entusiasmada acerca de una representación de Orfeo en Madrid, a cargo de la Compañía Caracol dirigida por Cipriano Rivas Cherif. Lo interesante de esta reseña es que Chacel destaca en Cocteau la conjunción de la vanguardia artística y del sentir religioso, sublimados por un valor clave: la belleza: "Una teoría religiosa puede dejar de ser fondo de la filosofía vigente, norma del fluir vital, y seguir siendo raudal de belleza. [...] ahora el arcángel Cocteau saluda a mademoiselle 
Marie. El catolicismo ha subido al cielo" (ibidem, 236). En el prefacio a la traducción de dos obras de Cocteau, Antígona y Reinaldo y Armida, Chacel va a volver sobre estos aspectos, esbozando el desarrollo de Cocteau como artista y como pensador sobre el arte que en Le Coq et L'Arlequin defiende el necesario equilibrio entre instinto y método. Chacel deduce de allí que "Naturalmente, una disciplina que tienda a armonizar en el arte estas dos cosas conduce por fuerza a una moral" (Cocteau 1952, 11). A continuación hace referencia a la conocida carta de Cocteau a Jacques Maritain en la que Cocteau presenta la religión como una fuerza purificadora del arte contemporáneo. Las resonancias con la cosmovisión de la propia autora son evidentes:

[...] las palabras que acabo de transmitir son las palabras de
un poeta, son palabras llenas de atrevimiento, representan
una juventud de una fe y la fe de una juventud. Esto no es
un juego de palabras: es un testimonio de quien perteneció
a aquella juventud, de quien sabe cómo asentíamos los que
íbamos inmediatamente detrás y cómo nos reconocíamos
en aquella voz que tenía el derecho de hablar por todos
(ibidem, 14).

Identificándose con estas palabras, la autora se afirma como parte integrante de esta vanguardia literaria que enaltecía valores religiosos y morales positivos, en contra de la vanguardia dominante que produce artilugios "asentados sobre una sólida pavimentación de moral atea" (ibídem, 15). Concluye que "Estas dos heroínas de Cocteau: Antigona, la santa, y Armida la hechicera, son como refugios de la esperanza, como noticias de una realidad traspasada por lo sobrenatural -realidad íntegra - que desmiente el mal" (ibídem, 17). En el fondo, la gran novela de Chacel, La sinrazón, no trata sino de esta misma lucha contra el mal en una realidad "traspasada por lo sobrenatural". 
Rosa Chacel se movía entre distintos géneros literarios, básicamente la novela y el ensayo pero también la poesía y se observa una íntima coherencia en toda su obra, resultado de su ambición de presentar una trayectoria que siguiese una línea recta desde la vanguardia de las primeras décadas del siglo XX hasta el presente. En este conjunto se insertan las traducciones asumidas, como la del ensayo de Renato Poggioli sobre el arte de vanguardia, encargada por la Revista de Occidente, algo que queda patente en este apunte de su diario:

Empecé la traducción con el mayor entusiasmo: me parecía que sacarla en el otoño, al año, más o menos, de haber aparecido el libro sobre el Ultraísmo, de Gloria Videla, y poder dar poco después mi libro de ensayos sobre la crisis del 20 al 30, era hacer algo coherente, que tal vez llegase a ser medianamente entendido. (22 de julio de 1963, Chacel 1982, 344).

El libro de Videla, El Ultraísmo (Estudios sobre movimientos poéticos de vanguardia en España) se había publicado en 1963, el libro de ensayos era Saturnal y la traducción conectaría, pues, su obra ensayística y novelesca con el arte de la vanguardia que sentía como su lugar en la literatura contemporánea.

\section{Conclusión}

En la trayectoria de Rosa Chacel, la relación entre traducción, exilio y dificultades económicas es evidente. Antes de los años cuarenta no encontramos ninguna traducción publicada y a partir de los años sesenta, cuando la escritora empieza a tener otro tipo de ingresos, ya no publica casi ninguna traducción.

Algunas traducciones las hizo por amistad (Libertad o muerte de Nikos Kazantzakis, Museo de cámara de Walmir Ayala), otras 
por necesidad económica (las obras de teatro), otras por interés personal (Racine, Cocteau, Poggioli) pero siempre tuvo cuidado de que el nivel literario de los originales fuese indiscutible. $\mathrm{Su}$ autoexigencia ha dado sus frutos, prueba de ello es que sus traducciones teatrales se siguen representando ${ }^{6}$. La aventura de la traducción queda documentada en su diario y se convierte en materia novelesca en dos obras con una carga autobiográfica notable: La sinrazón y Ciencias naturales. También con referencia a las traducciones de Rosa Chacel se podría citar a la autora en una carta a Ana María Moix: "[...] mis cosas son una sola cosa” (Chacel \& Moix 1998, 27).

${ }^{6}$ En 2001, Miguel Narros montó La dama no es para la hoguera de Christopher Fry para el Teatro Principal de Alicante (Martínez 2001). Joan Ollé escenificó Fedra en la traducción de Rosa Chacel, adaptada por Eduardo Mendoza y Pedro Gimferrer en 2004 (Torres 2004). 


\section{Referencias}

Chacel, Rosa. Prefacio. Antígona. Reinaldo y Armida. Por Jean Cocteau. Buenos Aires: Emecé, 1952.

. Timoteo Pérez Rubio y sus retratos del jardín. Madrid: Cátedra, 1980.

. Alcancía. Ida. Barcelona: Seix Barral, 1982.

. Ciencias naturales. Barcelona: Seix Barral, 1988.

. Obra completa 1. La sinrazón. Valladolid: Excma. Diputación de Valladolid. Centro de Estudios Literarios. Fundación Jorge Guillén, 1989.

- Obra completa 3. Artículos I. Valladolid: Excma. Diputación de Valladolid. Centro de Estudios Literarios. Fundación Jorge Guillén, 1993a.

- Obra completa 4. Artículos II. Valladolid: Excma. Diputación de Valladolid. Centro de Estudios Literarios. Fundación Jorge Guillén, 1993b.

Chacel, Rosa y Ana María Moix. De mar a mar. Epistolario Rosa Chacel-Ana María Moix. Prólogo, edición y notas de Ana Rodríguez Fischer. Barcelona: Península, 1998.

Cruces Colado, Susana. "Las traducciones de Camus en España durante el franquismo: difusión y censura." Transitions: Journal of Franco-Iberian studies 2 (2006): 82-113.

Flaubert, Gustave. L'éducation sentimentale. Paris: Garnier-Flammarion, 1969.

King, John. Sur. A study of the Argentine literary journal and its role in the development of a culture 1931-1970. Cambridge: Cambridge UP, 2009.

Lago Carballo, Antonio \& Nicanor Gómez-Villegas. Un viaje de ida y vuelta: la edición española e iberoamericano (1936-1975). Madrid: Siruela, 2006. 
Loedel Rois, Germán. Los traductores del exilio republicano español en Argentina [tesis inédita]. Barcelona: Universidad Pompeu Fabra, 2012. Red. 20 nov. 2015 $<$ http://repositori.upf.edu/handle/10230/20513 > .

Macciuci, Raquel. "La Guerra civil española en la Revista Sur." Sociohistórica 15-16 (2004): 29-63.

Martínez, Emilio. "Narros dirige en el Teatro Principal de Alicante una obra de Christopher Fry." El País, 30 oct. 2001: n.pag. Red.

Matamoro, Blas. "Chacel y Ayala: formas de eludir el exilio." Cuadernos Hispanoamericanos 714 (2009): 71-90.

Morán Rodríguez, Carmen. “ $\square U n$ escritor argentino’: Rosa Chacel, Identidad en Conflicto(s) y Estrategias de Inclusión." Gramma 24.50 (2013): 186-204.

Otero Carvajal, Luis E. La destrucción de la ciencia en España: depuración universitaria en el franquismo. Madrid: Editorial Complutense, 2006.

Pasternac, Nora. "La revista Sur y el exilio literario español." Estudios. Filosofía. Historia. Letras. ITAM México, 72 (2005) 7-19. Red. 18 nov. 2015. <http:// biblioteca.itam.mx/estudios/60-89/72/NoraPasternacLarevistasur.pdf $>$.

Rodríguez Fischer, Ana. "Rosa Chacel en su circunstancia." Rosa Chacel: premio nacional de las letras españolas. Ed. Dónoan et al. Barcelona: Anthropos Editorial (1990): 35-50.

Rodríguez Fischer, Ana. "Reportaje biográfico de Rosa Chacel." Rosa Chacel: premio nacional de las letras españolas. Barcelona: Anthropos Editorial (1990): $107-124$.

Torres, Rosana. "La excepcional 'Fedra' de Racine, Joan Ollé, Mendoza y Gimferrer se estrena en castellano”. El País. 19 mayo 2004: n. pag. Red.

Willson, Patricia. La constelación del Sur. Traductores y traducciones en la literatura argentina del siglo XX. Buenos Aires: Siglo veintiuno editores Argentina, 2009. 


\section{Traducciones por Rosa Chacel}

Ayala, Walmir. Museo de cámara. Madrid: Xanela, 1986.

Camus, Albert. La peste. Buenos Aires: Emecé, 1948. [Reediciones: 1951, 1957, 1968, 1970, 1979, 1983, 1988, 1990, 1994, 1995, 2005, 2006, 2010].

Cocteau, Jean. Antígona. Trad. Miguel Alfredo Olivera; Reinaldo y Armida. Trad. Rosa Chacel. Buenos Aires: Emecé, 1952.

Elliot, T.S. Reunión de familia. Prólogo Patrick O. Dudgeon. Buenos Aires: Emecé, 1953.

Fry, Christopher. La dama no es para la hoguera. Buenos Aires: Editorial Sudamericana, 1955.

. Venus observada. Buenos Aires: Editorial Sudamericana, 1955.

Holthusen, Hans Egon. Rainer María Rilke. Traducción de Rosa Chacel y Nicolás Wendkheim. Buenos Aires: La Mandrágora, 1960.

Kazantzakis, Nikos. Libertad o muerte. Buenos Aires: Carlos Lohlé, 1957.

Mallarmé, Stéphane. Herodías, en Ciclón. Revista literaria, La Habana, III, 2 (1957) 18-25. [Incluye la Escena, la Obertura antigua y un Canto del Bautista].

Noailles, Condesa Anna de. Una carta de las que no se envían. Buenos Aires: Arturo Jacinto Álvarez, 1948.

Poggioli, Renato. Teoría del arte de vanguardia. Madrid: Revista de Occidente, 1964.

Priestley, J. B. Edén término; El retamal; Cornelius, traducción de Rosa Chacel y Vera Macarow, Buenos Aires: Editorial Sudamericana, 1957. 
Racine, Jean. Tres tragedias. Buenos Aires: Sudamericana, 1958. [Contiene: Británico; Berenice; Bayaceto].

Racine, Jean. Seis tragedias. Madrid, Alfaguara, 1983. [Edición bilingüe, contiene: Andrómaca; Británico; Berenice; Bayaceto; Fedra; Atalía].

Vercors. Animales desnaturalizados. Buenos Aires: Imán, 1953.

Recebido em: 07/08/2017 Aceito em: 05/10/2017 Publicado em janeiro de 2018

Lieve Behiels é profesora de traducción especializada e interpretación en la Facultad de Letras (Campus de Amberes) de la KU Leuven. Sus campos de investigación son la literatura española de los siglos XIX y XX, las relaciones culturales entre España y los Países Bajos en los siglos XVI y XVII y los estudios históricos de traducción. En este campo, se ha dedicado a la traducción de la literatura espiritual y mística y ha participado en diversos proyectos relacionados con la traducción monacal. Es académica correspondiente para Bélgica de la RAE. Leuven, Flandes, Bélgica. E-mail: lieve.behiels@kuleuven.be 